\title{
SINGLE LOOP SPACE DECOMPOSITIONS
}

\author{
DAVID J. ANICK
}

\begin{abstract}
The method of single loop space decompositions, in which $\Omega X$ is factored into minimal factors, is an important one for understanding the unstable homotopy of many simply-connected spaces $X$. This paper begins with a survey of the major known theorems along these lines. We then give a necessary and sufficient condition for $\Omega X$ to be decomposable as a product of spaces belonging to a certain list. We conclude with a nontrivial instance of an application of this condition.
\end{abstract}

\section{BACKGROUND AND SUMMARY}

OF THE MAJOR KNOWN DECOMPOSITION THEOREMS

In this introduction we provide a summary of the major known theorems of a general nature regarding single loop space decompositions.

The original loop space decomposition was discovered by Hilton $[\mathrm{H}]$ in 1955 by building upon work of George Whitehead; it was later generalized by Milnor.

Theorem 1 (Hilton). Let $W$ be a simply-connected finite type wedge of spheres, i.e., $W=\bigvee_{i} S^{n_{i}}, n_{i} \geq 2$, and $n_{i} \rightarrow \infty$ if the index set is infinite. Then

$$
\Omega W \approx \prod_{j} \Omega S^{m_{j}},
$$

where $m_{j}=m_{j}\left(n_{1}, n_{2}, \ldots\right) \rightarrow \infty$.

Here, and throughout this article, $\approx$ denotes a weak homotopy equivalence for spaces, and an infinite product of pointed spaces is always assumed to denote the weak infinite product (i.e., the direct limit of all products over finite subsets of the index set).

Hilton utilized combinatorics and an explicit determination of the "basic products" in a free Lie algebra in order to prove Theorem 1. The principal interest of the theorem lies in its enabling us to reduce the problem of the homotopy groups of a bouquet of spheres to the problem of the unstable homotopy groups of spheres, along with a readily solvable combinatorial problem.

Another fact regarding loop spaces on spheres was recognized by Serre as early as 1951. In modern language, this fact is

Received by the editors October 10, 1990.

1980 Mathematics Subject Classification (1985 Revision). Primary 55P35, 55Q20.

The author was partially supported by NSF grant DMS-891-2842. 
Theorem 2 (Serre). Localized away from the prime 2,

$$
\Omega S^{2 m} \approx S^{2 m-1} \times \Omega S^{4 m-1}, \quad m \geq 1 .
$$

Notation/Definition. Let $\mathscr{S}$ denote the collection of pointed spaces, $\mathscr{S}=$ $\left\{S^{2 m-1}, \Omega S^{2 m+1} \mid m \geq 1\right\}$. For any collection $\mathscr{C}$ of pointed spaces, let $\prod_{0} \mathscr{C}$ denote the collection of finite type products all of whose factors belong to $\mathscr{C}$ (recall that any infinite product is automatically the weak infinite product), and let $\Pi \mathscr{C}$ denote the collection of spaces having the weak homotopy type of some member of $\prod_{0} \mathscr{C}$. It is obvious that $\prod\left(\prod \mathscr{C}\right)=\prod \mathscr{C}$.

Combining Theorems 1 and 2, we find

Theorem 3 (Hilton-Serre). Localized away from 2,

$$
\Omega\left(\bigvee_{i} S^{n_{i}}\right) \in \prod \mathscr{S}
$$

for any simply-connected finite type wedge of spheres $\bigvee_{i} S^{n_{i}}$.

There is a rather strong generalization of Theorem 3. Although it was probably known and used before 1981, its first appearance in the published literature was in Baues' book [B] of that year, and I will refer to it as the Hilton-SerreBaues (H-S-B) theorem.

Notation. Henceforth, $p$ denotes an odd prime, and $X$ always denotes a simplyconnected CW complex of finite type. Also, let

$$
h: \pi_{m}() \otimes \mathbb{Z}_{(p)} \rightarrow H_{m}\left(; \mathbb{Z}_{(p)}\right)
$$

denote the $p$-local Hurewicz homomorphism. Later we shall also encounter the Hurewicz homomorphism for mod $p$ homotopy,

$$
h: \pi_{m}\left(; \mathbb{Z}_{p}\right) \rightarrow H_{m}\left(; \mathbb{Z}_{p}\right) .
$$

Theorem 4 (H-S-B). Suppose the following conditions are satisfied:

(i) $H_{*}\left(\Omega X ; \mathbb{Z}_{(p)}\right)$ is free as a $\mathbb{Z}_{(p)}$-module, i.e., torsion-free; and

(ii) $H_{*}\left(\Omega X ; \mathbb{Z}_{(p)}\right)$ is generated as a $\mathbb{Z}_{(p)}$-algebra (via Pontrjagin multiplication) by $\operatorname{im}(h)$.

Then, localized at $p$, we have $\Omega X \in \prod \mathscr{S}$.

Baues' proof utilized the Poincaré-Birkhoff-Witt theorem to split the Pontrjagin ring algebraically, and then he realized the homotopy equivalence geometrically by multiplying together a corresponding list of maps into $\Omega X$. Because he was in the context of working with Lie algebras, Baues required $p \geq 5$; the author gave a similar proof, valid for any odd $p$, in [A2].

The H-S-B theorem does apply to a great many spaces, such as arbitrary subcomplexes of a product of spheres (provided this product has the standard $\mathrm{CW}$ structure), and to some examples having higher Massey products like $\left(S^{3} \vee\right.$ $\left.S^{3}\right) \cup \cup_{\left[l_{1},\left[l_{1}, t_{2}\right]\right]} e^{8}$.

Unfortunately, condition (i) fails for many spaces; there are even finite spaces $X$ for which (i) fails to hold at any $p$ [A1]. Furthermore, there is no obvious way to check condition (ii) in general, especially since the Pontrjagin ring will typically not be finitely generated. One case that has been settled is 
Theorem 5 [A2]. Let $X$ be a two-cone, i.e., the cofiber in

$$
\bigvee_{i} S^{n_{i}} \rightarrow \bigvee_{j} S^{m_{j}} \rightarrow X
$$

Suppose $p$ lies outside a certain set of primes that depends upon $X$ (this set will be finite if $X$ is finite). If condition (i) of Theorem 4 holds for $X$, then condition (ii) holds also.

McGibbon and Wilkerson [MW] first introduced the approach, illustrated in Theorem 5 above, of disregarding behavior at a finite set of exceptional primes, concentrating instead on results that might hold for the "generic" prime. They proved

Theorem 6 (Wilkerson-McGibbon). Suppose $X$ is finite and rationally elliptic, i.e., $\operatorname{dim}_{\mathbb{Q}}\left(\pi_{*}(X) \otimes \mathbb{Q}\right)<\infty$. Then, at almost all primes $p, \Omega X \in \prod \mathscr{S}$.

There is only so far one can go with the torsion-free spaces in $\prod \mathscr{S}$, however. Most loop spaces do have torsion in their homology at various primes, and often we are most interested in the homotopy at a prime where interesting torsion occurs. The theory of loop space decompositions for torsion spaces seems at first to be quite different from the H-S-B theorem and its extensions.

The remainder of this section will be concerned with torsion. The principal result in this area is the work of Cohen-Moore-Neisendorfer (C-M-N) on the loops on the Moore space.

Notation. If $[k]$ denotes the self-map on $S^{m}$ of Brouwer degree $k$, then $P^{m+1}(k)$ is the homotopy-theoretic cofiber of $[k]$, and $S^{m}\{k\}$ is the homotopytheoretic fiber of $[k]$.

Cohen, Moore, and Neisendorfer constructed a family of $p$-torsion spaces, denoted $T^{2 m+1}\left\{p^{r}\right\}, m \geq 1, r \geq 1$, having the following properties:

$$
\begin{aligned}
& \Omega P^{2 m+2}\left(p^{r}\right) \approx S^{2 m+1}\left\{p^{r}\right\} \times \Omega W^{2 m+2}, \\
& \Omega P^{2 m+1}\left(p^{r}\right) \approx T^{2 m+1}\left\{p^{r}\right\} \times \Omega W^{2 m+1},
\end{aligned}
$$

where each $W^{j}$ is a certain $(2 j-4)$-connected wedge of $\bmod p^{r}$ Moore spaces; $\bar{H}_{*}\left(S^{2 m+1}\left\{p^{r}\right\}\right)$ and $\bar{H}_{*}\left(T^{2 m+1}\left\{p^{r}\right\}\right)$ are all $\mathbb{Z}_{p^{r} \text {-torsion; there is a fibration }}$ sequence

$$
S^{2 m-1} \times \prod_{j=1}^{\infty} S^{2 m p^{j}-1}\left\{p^{r+1}\right\} \rightarrow T^{2 m+1}\left\{p^{r}\right\} \rightarrow \Omega S^{2 m+1},
$$

whose cohomology Serre spectral sequence over $\mathbb{Z}_{p}$ degenerates; and $p^{r+1}$ is a homotopy exponent, i.e., $p^{r+1} \cdot \pi_{*}\left(T^{2 m+1}\left\{p^{r}\right\}\right)=0$ [N2].

By applying (2) and (3) and the Hilton-Milnor theorem iteratively, one obtains

Theorem 7 (C-M-N). Let $\mathscr{T}^{\prime}$ denote the collection of spaces $T^{2 m+1}\left\{p^{r}\right\}$ and $S^{2 m+1}\left\{p^{r}\right\}$ for $r \geq 1$ and $m \geq 1$. Let $\left\{r_{i}\right\}_{i \in I}$ and $\left\{m_{i}\right\}_{i \in I}$ be countably indexed sets such that $r_{i} \geq 1$ and $m_{i} \geq 3$, and either $I$ is finite or $m_{i} \rightarrow \infty$. Then

$$
\Omega\left(\bigvee_{i \in I} P^{m_{i}}\left(p^{r_{i}}\right)\right) \in \prod^{\mathscr{T}^{\prime}}
$$


The spaces $S^{2 m+1}\left\{p^{r}\right\}$ are atomic. In [CMN2] Cohen, Moore, and Neisendorfer demonstrate that the spaces $T^{2 m+1}\left\{p^{r}\right\}$ are atomic with the single remarkable exception that $T^{3}\{p\} \approx T^{2 p+1}\{p\} \times U^{1}$ for a certain atomic space $U^{1}$. Recall [CMN2] that a space $X$ is called atomic if (for some $d$ ) it is $(d-1)$ connected, $\pi_{d}(X)$ is a cyclic abelian group, and every self-map $f: X \rightarrow X$ inducing an isomorphism on $\pi_{d}()$ is a homotopy equivalence. Let $\mathscr{T}$ consist of those spaces in $\mathscr{T}^{\prime}$ which are atomic (i.e., all of $\mathscr{T}^{\prime}$ except for $T^{3}\{p\}$ ) together with the singleton $U^{1}$. Then it is clear that $(5)$ can be replaced by

$$
\Omega\left(\bigvee_{i \in I} P^{m_{i}}\left(p^{r_{i}}\right)\right) \in \prod \mathscr{T} .
$$

In view of Theorems 3 and 7, the smallest collection of atomic spaces into which a general $p$-local loop space might conceivably be decomposable is the union $\mathscr{S} \cup \mathscr{T}$. This decomposition does hold for any space $X$ which is a wedge of spheres and mod $p^{r_{i}}$ Moore spaces. Experiment reveals that a typical finite complex has a few small primes at which such a decomposition fails (i.e., more types of factors are needed), but the set $\mathscr{S} \cup \mathscr{T}$ suffices at all large enough primes. Thus the following conjecture is ambitious but not impossible.

Conjecture. Let $X$ be finite. Then, at almost all primes $p$,

$$
\Omega X \in \prod(\mathscr{S} \cup \mathscr{T})
$$

This conjecture may be viewed as the motivation behind the results of $\S 2$. It has many immediate consequences, including Moore's conjecture [NS] at large primes and a revised version of Wilkerson's conjecture [MW] asserting the triviality of the Steenrod operations $\mathscr{P}^{i}$ on $H^{*}\left(\Omega X ; \mathbb{Z}_{p}\right)$ for large $p$.

\section{A NECESSARY AND SUFFICIENT CONDITION FOR $\Omega X \in \prod(\mathscr{S} \cup \mathscr{T})$}

In this section we provide an alternate, more elegant proof for the HiltonSerre-Baues theorem. This new proof can be generalized to permit $p$-torsion, and we obtain a necessary and sufficient condition for $\Omega X$ to belong to $\Pi(\mathscr{S} \cup$ $\mathscr{T})$. The condition is expressed in terms of multiplicative generators for the successive algebras in the homology Bockstein spectral sequence for $\Omega X$.

We begin by observing that each space belonging to $\mathscr{S} \cup \mathscr{T}$ is an $H$-space, since each is a factor of a loop space. The following theorem, a slight generalization of one proved in [W], is clearly relevant.

Theorem 8 (Wilkerson). Let $\mathscr{C}$ denote any collection of atomic $H$-spaces. If $X \in \Pi \mathscr{C}$ and $Y$ is a retract of $X$, then $Y \in \prod \mathscr{C}$.

We also note the following lemma, whose proof we deem obvious.

Lemma A. If $W$ is 1-connected finite type wedge of spheres, $Z$ is a 1-connected, and $f: W \rightarrow Z$ induces a split surjection on $H_{*}\left(; \mathbb{Z}_{(p)}\right)$, then $Z$ is p-locally $a$ wedge of spheres.

New proof of the $H-S-B$ theorem (Theorem 4). Let $\bigvee S^{m_{i}}=W \stackrel{g}{\rightarrow} \Omega W$ surject to $Q H_{*}\left(\Omega X ; \mathbb{Z}_{(p)}\right)$, where $Q$ denotes the module of indecomposables. Such a map $g$ exists because of condition (ii). There is a unique extension of $g$ to an $H$-map from the James construction on $W$ to $\Omega X$, call it $\tilde{g}: \Omega \Sigma W \rightarrow \Omega X$. 
Then $H_{*}\left(\tilde{g} ; \mathbb{Z}_{(p)}\right)$ is a surjection, and it is a split surjection by condition (i). Apply Lemma A to $\Sigma \tilde{g}: \Sigma \Omega \Sigma W \rightarrow \Sigma \Omega X$ to deduce that $\Sigma \Omega X$ is $p$-locally a wedge of spheres. Finally, $\Omega \Sigma \Omega X \in \prod \mathscr{S}$ by Theorem 3 , and $\Omega X$ is a retract of $\Omega \Sigma \Omega X$, so Theorem 8 yields $\Omega X \in \prod \mathscr{S}$.

To generalize this proof to allow for $p$-torsion, we recall the properties of the Bockstein spectral sequence (henceforth "BSS") for a connected space $Y$ of finite type [N1]. It is a homology spectral sequence $\left\{E^{r}=E^{r}(Y), \beta^{r}\right\}$ with $E^{1}=H_{*}\left(Y ; \mathbb{Z}_{p}\right)$ and $E^{\infty} \approx$ (free abelian component of $\left.H_{*}(Y)\right) \otimes \mathbb{Z}_{p}$. Each differential $\beta^{r}$ has degree -1 . If $\beta^{r}(y)=x$ then $y$ and $x$ represent $\mathbb{Z}_{p^{r}}$ torsion in $H_{|x|}(Y)$. There is a Hurewicz homomorphism

$$
h^{r}: E_{(\pi)}^{r} \rightarrow E^{r},
$$

where $E_{(\pi)}^{r}$ denotes the $r$ th term in an analogous homotopy Bockstein spectral sequence $(\pi \mathrm{BSS})$, for the mod $p$ homotopy groups of $Y$. Lastly, when $Y=$ $\Omega X$, each $\left(E^{r}, \beta^{r}\right)$ is a differential graded cocommutative Hopf algebra over $\mathbb{Z}_{p}$.

Notation. Write $X \in \mathscr{W}$ if $X$ has the weak $p$-local homotopy type of a 1connected finite type wedge of spheres and various mod $p^{r_{i}}$ Moore spaces.

The lemma generalizing Lemma $\mathrm{A}$ is

Lemma B. If $W \in \mathscr{W}, Z$ is 1-connected, and $f: W \rightarrow Z$ induces a surjection of BSS terms $E^{r}($ ) for all $r$ (including $r=\infty$ ), then $Z \in \mathscr{W}$.

Theorem 9. The following are equivalent.

(a) $\Omega X \in \prod(\mathscr{S} \cup \mathscr{T})$.

(b) $\Sigma \Omega X \in \mathscr{W}$.

(c) For each $r \geq 1$ and for $r=\infty$, the BSS term $E^{r}(\Omega X)$ is generated as a $\mathbb{Z}_{p}$-algebra by $\operatorname{im}\left(h^{r}: E_{(\pi)}^{r}(\Omega X) \rightarrow E^{r}(\Omega X)\right)$.

Proof. That (a) implies (b) is easy. Each space belonging to $\mathscr{S} \cup \mathscr{T}$ is a retract of a space whose suspension lies in $\mathscr{W}$, so $\Sigma Y \in \mathscr{W}$ if $Y \in \mathscr{S} \cup \mathscr{T}$. Next, the collection $\mathscr{W}$ is closed under wedges and smash products. Hence the collection of spaces $Y$ for which $\Sigma Y \in \mathscr{W}$ is closed under products. In particular, this collection contains $\prod_{0}(\mathscr{S} \cup \mathscr{T})$.

To prove (b) implies (a), first note by Theorems 3 and 7 and the HiltonMilnor theorem that $Y \in \mathscr{W}$ implies $\Omega Y \in \prod(\mathscr{S} \cup \mathscr{T})$. Thus $\Omega \Sigma \Omega X \in$ $\prod(\mathscr{S} \cup \mathscr{T})$. Now $\Omega X$ is a retract of $\Omega \Sigma \Omega X$; apply Theorem 8 .

To prove (b) implies (c), recall that the retraction from $\Omega \Sigma \Omega X$ to $\Omega X$ is actually the map $\Omega e$, where $e: \Sigma \Omega X \rightarrow X$ is the evaluation of a loop at a suspension coordinate. Since $\Omega e$ is an $H$-map, the homomorphism that it induces on the $E^{r}$ term of the BSS is a homomorphism of $\mathbb{Z}_{p}$-algebras. Moreover, coming from a retraction, this homomorphism is a surjection, and induces a surjection of indecomposable modules. Thus it suffices to check (using the naturality of $\left.h^{r}\right)$ that each of the terms $E^{r}(\Omega \Sigma \Omega X)$ is generated as an algebra by Hurewicz images. This holds because whenever $Y$ is a suspension, then $E^{r}(\Omega Y)$ is the free $\mathbb{Z}_{p}$-algebra on the reduced desuspension of $E^{r}(Y)$. If $Y=\Sigma \Omega X \in \mathscr{W}$ and we write $Y \approx \Sigma W$ with $W \in \mathscr{W}$, then the adjoint map $W \rightarrow \Omega Y$ carries $E^{r}(W)$, which consists entirely of Hurewicz images, to the algebra generators of $E^{r}(\Omega Y)$. 
Finally, let us show that (c) implies (b). Assuming (c), we may construct maps $f^{r}: W^{r} \rightarrow \Omega X$ for which $W^{r} \in \mathscr{W}$ and $E^{r}\left(f^{r}\right)$ surjects onto $Q\left(E^{r}(\Omega X)\right)$. The connectivity of $W^{r}$ goes to infinity as $r \rightarrow \infty$ (although $W^{\infty}$ corresponding to $r=\infty$ will not fit this pattern), so put $W=\left(\bigvee_{r=1}^{\infty} W^{r}\right) \vee W^{\infty} \in \mathscr{W}$. Observe that $f=\left(\bigvee_{r=1}^{\infty} f^{r}\right) \vee f^{\infty}: W \rightarrow \Omega X$ surjects on $Q E^{r}()$ for all $r$ simultaneously. The James construction on $W$ permits the construction of the $H$-map $\tilde{f}: \Omega \Sigma W \rightarrow \Omega X$, which surjects on $E^{r}()$ for all $r$. By Lemma $\mathrm{B}$, $\Sigma \Omega X \in \mathscr{W}$, since $\Sigma \Omega \Sigma W \in \mathscr{W}$. This completes the proof.

Remark. Theorem 9 provides a plausible approach to proving the Conjecture. If at large enough primes each term of the BSS for $\Omega X$ can be shown to be generated by Hurewicz images, then the Conjecture will be proved. Conversely, if for some specific finite $X$ there are (at infinitely many primes) BSS terms requiring non-Hurewicz generators, then the Conjecture is false.

\section{COMPUTATION FOR A SPECIFIC SPACE}

In this section we examine in detail the BSS for a specific three-cell space $K$. We show that $\Omega K$ does satisfy condition (c) of Theorem 9, and consequently that $\Omega K$ belongs to $\Pi(\mathscr{S} \cup \mathscr{T})$. In the course of the computation, we illustrate some techniques that may be useful for verifying condition (c) in general. We summarize our conclusions, and we discuss the importance of $K$.

We assume henceforth that $p \geq 5$. The space we shall consider is $K=$ $P^{2 n+1}\left(p^{r}\right) \cup_{[l, l]} e^{4 n}$, where the attaching map is the Whitehead square of the identity on the $(2 n)$-cell. Alternatively, $K$ may be viewed as the push-out over $S^{2 n}$ of $P^{2 n+1}\left(p^{r}\right)$ and the second stage $J_{2}\left(S^{2 n}\right)$ of the James construction on $S^{2 n}$ :

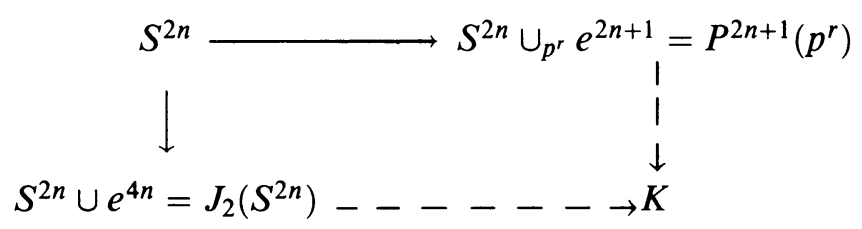

We will verify condition (c) of Theorem 9 . It will turn out that there are just two nonzero differentials in the BSS for $\Omega K$, namely $\beta^{r}$ and $\beta^{2 r}$. We will first determine the entire BSS, and then explain why each algebra in the sequence is generated by Hurewicz images.

Our principal tool will be the Adams-Hilton model [AH, A3] for $K$. The view (7) of $K$ as a push-out tells us immediately that a model is the associative differential graded algebra (henceforth "dga")

$$
\begin{gathered}
(A, d)=(\mathbb{Z}\langle a, b, c\rangle, d), \quad|a|=2 n-1, \quad|b|=2 n,|c|=4 n-1, \\
d(a)=0, \quad d(b)=p^{r} a, \quad d(c)=2 a^{2} .
\end{gathered}
$$

Our first task is to compute the $\bmod p \operatorname{BSS}$ for $(A, d)$, which will coincide with the BSS for $\Omega K$.

The $E^{1}$ term is $H_{*}\left(A \otimes \mathbb{Z}_{p}, d \otimes \mathbb{Z}_{p}\right)$. Keeping the same notation $a, b, c$ for these elements' mod $p$ reductions, we find that the dga is now

$$
\mathbb{Z}_{p}\langle a, b, c\rangle, \quad d(a)=0=d(b), \quad d(c)=2 a^{2},
$$

isomorphic with $\left(\mathbb{Z}_{p}\langle a, c\rangle, d\right) \amalg\left(\mathbb{Z}_{p}[b], 0\right)$. The dga $\left(\mathbb{Z}_{p}\langle a, c\rangle, d(c)=2 a^{2}\right)$ is well known, e.g., it is the $\mathbb{Z}_{p}$-Adams-Hilton model for $J_{2}\left(S^{2 n}\right)$. Its homology 
is the commutative ring $B=\mathbb{Z}_{p}[\mathbf{a}, \mathbf{e}] /\left(\mathbf{a}^{2}\right)$, where $\mathbf{a}$ is the homology class of the cycle $a$ and $\mathbf{e}$ is the class of $e$, where $e$ equals the (graded) commutator of $a$ and $c, e=[a, c]=a c+c a$. We will henceforth write $a$ and $e$ for a and $\mathbf{e}$.

The fact that $a$ and $e$ are cycles in the $\mathbb{Z}$-dga $(A, d)$ means that no Bockstein differentials can originate on them, nor on elements of the subalgebra they generate. Since $E^{1}=B \amalg \mathbb{Z}_{p}[b]$ is generated by the set $\{a, e, b\}$, and $\beta^{i}$ vanishes on each of these for $i<r$, we have $E^{r}=E^{1}$. However, $\beta^{r}(a)=$ $\beta^{r}(e)=0$ with $\beta^{r}(b)=a$, so to obtain the $E^{r+1}$ term we must compute the homology of $\left(B \amalg \mathbb{Z}_{p}[b], \beta^{r}\right)$.

The computation of this homology algebra is not a trivial task. We must rely upon various techniques for working with dga's. We first replace $B$ by a model, i.e., a free dga $(C, \gamma)$ together with a quism from $(C, \gamma)$ to $(B, 0)$. In this case, we have the good fortune that $B$ equals the Ext algebra of a commutative ring, specifically, of $S=\mathbb{Z}_{p}[\alpha, \varepsilon] /\left(\varepsilon^{2}\right)$, so the cobar construction on $S$ is a good candidate for $(C, \gamma)$. Put

$$
\begin{array}{cc}
C=\mathbb{Z}_{p}\left\langle a_{1}, a_{2}, \ldots, e_{0}, e_{1}, \ldots\right\rangle, \quad\left|a_{j}\right|=2 n j-1,\left|e_{j}\right|=6 n-2+2 n j, \\
\gamma\left(a_{j}\right)=-\sum_{i=1}^{j-1} a_{i} a_{j-i}, \quad & \gamma\left(e_{j}\right)=\sum_{i=0}^{j-1}\left[e_{i}, a_{j-i}\right] .
\end{array}
$$

The quism $\lambda:(C, \gamma) \rightarrow(B, 0)$ is simply the quotient homomorphism that divides out by the ideal generated by $\left\{a_{1}^{2}, a_{2}, a_{3}, \ldots,\left[a_{1}, e_{0}\right], e_{1}, e_{2}, \ldots\right\}$.

It does not follow automatically that

$$
\lambda \amalg 1:\left(C \amalg \mathbb{Z}_{p}[b], \delta\right) \rightarrow\left(B \amalg \mathbb{Z}_{p}[b], \beta^{r}\right)
$$

is a quism, where $\left.\delta\right|_{C}=\gamma$ and $\delta(b)=a_{1}$. To prove that it is a quism, we employ a spectral sequence argument. Let $D=C \amalg \mathbb{Z}_{p}[b]$, and bigrade $D$ by letting $b, a_{i}$, and $e_{i}$ have bigrades $(1,0),(0, i-1)$, and $(0, i)$, respectively. $\left(D_{* *}, \delta\right)$ becomes a double complex when we write $\delta=\delta_{0}+\delta_{1},\left.\delta_{0}\right|_{C}=\gamma$, $\delta_{0}(b)=0, \delta_{1}(C)=0, \delta_{1}(b)=a_{1}$; notice that $\delta_{0}\left(B_{s t}\right) \subseteq B_{s, t-1}$ and $\delta_{1}\left(B_{s t}\right) \subseteq$ $B_{s-1, t}$. Associated to this double complex is a spectral sequence $\left\{E^{q}(D), \delta^{q}\right\}$ converging to $H_{*}(D, \delta)$. Computing, we find that

$$
E^{1}(D)=H_{*}\left(D, \delta_{0}\right)=H_{*}(C, \gamma) \amalg \mathbb{Z}_{p}[b]=B \amalg \mathbb{Z}_{p}[b],
$$

and that the differential $\delta^{1}$ on $E^{1}(D)$ may be identified with $\beta^{r}$. Thus

$$
E^{r+1}(A)=H_{*}\left(B \amalg \mathbb{Z}_{p}[b], \beta^{r}\right)=H_{*}\left(E^{1}(D), \delta^{1}\right)=E^{2}(D),
$$

while $E^{\infty}(D)=H_{*}(D, \delta)$. But $E^{1}(D)$ is generated by the elements $\{a, b, e\}$, which come from $\left\{a_{1}, b, e_{0}\right\}$, which all lie on the 0th row of the spectral sequence. Since $\delta^{q}$ has bidegree $(-q, q-1)$, it must vanish for $q \geq 2$, whence $E^{\infty}(D)=E^{2}(D)$, and (9) is indeed a quism.

The fact that (9) is a quism is useful because we can now compute $H_{*}(D, \delta)$ in an entirely different way. The generators $b$ and $a_{1}$ of $D$ clearly "cancel," e.g., the quotient homomorphism

$$
(D, \delta) \rightarrow(G, \theta),
$$


where $G$ is the quotient of $D$ by the ideal generated by $a_{1}$ and $b$, is another quism. Clearly,

$$
\begin{gathered}
G=\mathbb{Z}_{p}\left\langle a_{2}, a_{3}, \ldots, e_{0}, e_{1}, \ldots\right\rangle, \quad\left|a_{j}\right|=2 n j-1,\left|e_{i}\right|=6 n-2+2 n j, \\
\theta\left(a_{j}\right)=-\sum_{i=2}^{j-2} a_{i} a_{j-i}, \quad \theta\left(e_{j}\right)=\sum_{i=0}^{j-2}\left[e_{i}, a_{j-i}\right] .
\end{gathered}
$$

We may recognize $G$ as the cobar construction on the subring

$$
R=\mathbb{Z}_{p}\left(1, \alpha^{2}, \alpha^{3}, \ldots, \varepsilon, \alpha \varepsilon, \alpha^{2} \varepsilon, \ldots\right)
$$

(where $\varepsilon^{2}=0$ ) of $S$, so $H_{*}(G, \theta)$ coincides (after a dimension shift) with $\operatorname{Ext}_{R}\left(\mathbb{Z}_{p}, \mathbb{Z}_{p}\right)$ as a $\mathbb{Z}_{p}$-algebra. In order to compute $E^{r+1}(A)$ we have been led to study the Ext algebra of $R$. Notice that $R$ is isomorphic as a ring with the quotient of a polynomial ring,

$$
\begin{aligned}
R & =\mathbb{Z}_{p}\left[\alpha_{2}, \alpha_{3}, \varepsilon_{0}, \varepsilon_{1}\right] /\left(\alpha_{2}^{3}-\alpha_{3}^{2}, \varepsilon_{0} \alpha_{3}-\varepsilon_{1} \alpha_{2}, \varepsilon_{0} \alpha_{2}^{2}-\varepsilon_{1} \alpha_{3}, \varepsilon_{0}^{2}, \varepsilon_{0} \varepsilon_{1}, \varepsilon_{1}^{2}\right) \\
& =\left(\left(\mathbb{Z}_{p}\left[\alpha_{2}, \alpha_{3}\right] /\left(\alpha_{2}^{3}-\alpha_{3}^{2}\right)\right) \otimes \mathbb{Z}_{p}\left[\varepsilon_{0}, \varepsilon_{1}\right] /\left(\varepsilon_{0}^{2}, \varepsilon_{0} \varepsilon_{1}, \varepsilon_{1}^{2}\right)\right) /\left(\varepsilon_{0} \alpha_{3}-\varepsilon_{1} \alpha_{2}, \varepsilon_{0} \alpha_{2}^{2}-\varepsilon_{1} \alpha_{3}\right) .
\end{aligned}
$$

We want the Ext algebra for the commutative Noetherian ring $R$, and such Ext algebras are notoriously difficult to compute. However, a presentation for the subalgebra of $\operatorname{Ext}_{R}\left(\mathbb{Z}_{p}, \mathbb{Z}_{p}\right)$ generated by $\operatorname{Ext}_{R}^{1}\left(\mathbb{Z}_{p}, \mathbb{Z}_{p}\right)$, call it $\left\langle\operatorname{Ext}_{R}^{1}\right\rangle$, may be written down immediately, since its presentation is obtained by "dualizing" the quadratic terms of the relators in the presentation (10) for $R$. Specifically,

$$
\begin{aligned}
\left\langle\operatorname{Ext}_{R}^{1}\right\rangle & =\mathbb{Z}_{p}\left\langle u_{2}, u_{3}, v_{0}, v_{1}\right\rangle /\left\langle u_{2}^{2},\left[u_{2}, u_{3}\right],\left[u_{2}, v_{0}\right],\left[u_{2}, v_{1}\right]+\left[u_{3}, v_{0}\right]\right\rangle \\
& =\mathbb{Z}_{p}\left\langle u_{3}, v_{0}, v_{1}\right\rangle \odot\left(\mathbb{Z}_{p}\left[u_{2}\right] /\left(u_{2}^{2}\right)\right) .
\end{aligned}
$$

Here $\odot$ denotes the semitensor product $[\mathrm{Sm}]$ of the Hopf algebra $\mathbb{Z}_{p}\left[u_{2}\right] /\left(u_{2}^{2}\right)$ acting on the left on the free algebra $\mathbb{Z}_{p}\left\langle u_{3}, v_{0}, v_{1}\right\rangle$. The action is determined by the information that $u_{2}$ acts trivially on $u_{3}$ and on $v_{0}$, while it takes $v_{1}$ to $-\left[u_{3}, v_{0}\right]$.

Having computed $\left\langle\operatorname{Ext}_{R}^{1}\right\rangle$, we now make the assertion that the subalgebra $\left\langle\operatorname{Ext}_{R}^{1}\right\rangle$ actually equals the whole algebra $\operatorname{Ext}_{R}\left(\mathbb{Z}_{p}, \mathbb{Z}_{p}\right)$. By [BF] this assertion is equivalent to the relationship between their Hilbert series,

$$
H_{R}(t)^{-1}=H_{\left\langle\mathrm{Ext}_{R}^{1}\right\rangle}(-1, t)
$$

whenever $R$ has an internal grading that renders it dimension-wise finite. Actually, our ring $R$ has two independent gradings: let the generators $\alpha_{2}, \alpha_{3}, \varepsilon_{0}$, and $\varepsilon_{1}$ have the bidegrees $(2,0),(3,0),(1,0)$, and $(1,1)$, respectively. Then, in addition to its homological grading, $\left\langle\operatorname{Ext}_{R}^{1}\right\rangle$ inherits the bigrading given by: $u_{2}-(2,0) ; u_{3}-(3,0) ; v_{0}-(1,0) ;$ and $v_{1}-(1,1)$. In the presence of a bigrading, the criterion (13) becomes

$$
H_{R}(s, t)^{-1}=H_{\left\langle\operatorname{Ext}_{R}^{1}\right\rangle}(-1, s, t) .
$$

Let us check (14). The Hilbert series of $R$ is easily seen to be

$$
H_{R}(s, t)=1+\frac{s^{2}}{1-s}+\frac{t}{1-s}=\frac{1-s+s^{2}+t}{1-s},
$$


while for $\left\langle\operatorname{Ext}_{R}^{1}\right\rangle$ it is ( $\lambda$ keeps track of the homological grading, and $s$ and $t$ the internal grading; use (12) to compute):

$$
H_{\left\langle\operatorname{Ext}_{R}^{1}\right\rangle}(\lambda, s, t)=\frac{1+\lambda s^{2}}{1-\lambda t-\lambda s t-\lambda s^{3}} .
$$

Sure enough, setting $\lambda=-1$ in (16) gives an expression whose reciprocal simplifies to (15). Deduce that $E^{r+1}(A)$ equals $H_{*}(D, \delta)$ equals $H_{*}(G, \theta)$ equals $\operatorname{Ext}_{R}\left\langle\mathbb{Z}_{p}, \mathbb{Z}_{p}\right\rangle$ equals $\left\langle\operatorname{Ext}_{R}^{1}\right\rangle$ equals the algebra given in (11).

Let us attempt to understand this algebra better. Tracing the relationships through, we find that the isomorphism between (11) and $E^{r+1}$ carries the generators $u_{2}, u_{3}, v_{0}$, and $v_{1}$ to the BSS elements that survive from $p^{r} c-[b, a]$, $2[b,[b, a]]+3 p^{r}[c, b], e=[c, a]$, and $p^{r} c^{2}+2[[c, a], b]$, respectively, in $A$. We will adopt the notations $u_{2}, u_{3}, v_{0}$, and $v_{1}$ for these four elements of $A$ or of $E^{r+1}(A)$.

We easily compute that $u_{2}, v_{0}$, and $v_{1}$ are cycles in $A$, while $d\left(u_{3}\right)=$ $-3 p^{2 r} v_{0}$. Since all four of these generators of $E^{r+1}$ have vanishing $\beta^{s}$ for $r+1 \leq s<2 r$, we know that $E^{2 r}=E^{r+1}$. Then $\beta^{2 r}\left(u_{3}\right)=-3 v_{0}$, with $\beta^{2 r}\left(u_{2}\right)=\beta^{2 r}\left(v_{0}\right)=\beta^{2 r}\left(v_{1}\right)=0$.

Our next step is to determine $E^{2 r+1}=H_{*}\left(E^{2 r}, \beta^{2 r}\right)$. To do this, note that the semitensor product representation (12) for $E^{2 r}$ is compatible with the differential, since the differential commutes with the action and vanishes on the acting Hopf algebra $\mathbb{Z}_{p}\left[u_{2}\right] /\left(u_{2}^{2}\right)$. It follows that $H_{*}\left(E^{2 r}, \beta^{2 r}\right)$ equals the semitensor product of $\mathbb{Z}_{p}\left[u_{2}\right] /\left(u_{2}^{2}\right)$ with $H_{*}\left(\mathbb{Z}_{p}\left\langle u_{3}, v_{0}, v_{1}\right\rangle, \beta^{2 r}\right)=\mathbb{Z}_{p}\left[v_{1}\right]$. Thus,

$$
E^{2 r+1} \approx \mathbb{Z}_{p}\left[v_{1}\right] \odot\left(\mathbb{Z}_{p}\left[u_{2}\right] /\left(u_{2}^{2}\right)\right)=\mathbb{Z}_{p}\left[u_{2}, v_{1}\right] /\left(u_{2}^{2}\right) .
$$

As we have noted, the $u_{2}$ and $v_{1}$ in (17) originate on the integral cycles $u_{2}=$ $p^{r} c-[b, a]$ and $v_{1}=p^{r} c^{2}+2[[c, a], b]$ in $A$. Therefore $\beta^{s}$ vanishes for $s \geq 2 r+1$, i.e., $E^{\infty}=E^{2 r+1}$. We have computed the entire BSS for the dga $(A, d)$ which is also the BSS for $\Omega K$.

It remains only to check condition (c) of Theorem 9, i.e., that each of the generators we have found, for each term of the BSS, is indeed a Hurewicz image. We must cover all of these cases: $a(s \leq r), b(s \leq r), e(s \leq 2 r), u_{2}(s>r)$, $u_{3}(r<s \leq 2 r)$, and $v_{1}(s>r)$.

The terms $E^{s}$ for $1 \leq s \leq r$ are generated by the set $\{a, b, e\}$. Referring to (7), note that $a$ and $b$ lie in the $E^{s}(\Omega())$ image from $P^{2 n+1}\left(p^{r}\right)$, where they are clearly Hurewicz images. Let us denote by $\tilde{a}$ and $\tilde{b}$ the elements of $\pi_{*}\left(\Omega K ; \mathbb{Z}_{p^{r}}\right)$ for which $h^{s}(\tilde{a})=a$ and $h^{s}(\tilde{b})=b$. The element $e$ lies in the $E^{s}(\Omega())$ image from $J_{2}\left(S^{2 n}\right)$, where it is (except for a factor of 3 ) the Hurewicz image of the adjoint of the attaching map for the $6 n$-cell of $J_{3}\left(S^{2 n}\right)$. Actually, the latter argument is valid for all $s$, even for $s>r$; it shows that (the class of) $e$ in $E^{s}(\Omega K)$ is an $h^{s}$-image, say of $\tilde{e}$, and because $\tilde{e}$ comes from an integral homotopy class, $\beta_{(\pi)}^{s}(\tilde{e})=0$ for all $s$.

Consider next $u_{2}=[b, a]$, which is a generator of $E^{s}$ for $s \geq r+1$. Put $\tilde{u}_{2}=[\tilde{b}, \tilde{a}] \in \pi_{4 n-1}\left(\Omega K ; \mathbb{Z}_{p^{r}}\right)$. The composite

$$
S^{4 n-2} \rightarrow P^{4 n-1}\left(p^{r}\right) \stackrel{\tilde{u}_{2}}{\rightarrow} \Omega K
$$

vanishes (cf. (7)), so $\tilde{u}_{2}$ extends (via the pinch map) over $S^{4 n-1}$. It follows that $\tilde{u}_{2}$ reduces to an infinite cycle, call it $\tilde{u}$, in $E_{(\pi)}^{r}(\Omega K)$. The fact that $h^{r}(\tilde{u})=u_{2}$ 
survives to $E^{\infty}(\Omega K)$ means that $\tilde{u}$ survives to $E_{(\pi)}^{\infty}$. Let $u^{*} \in \pi_{4 n-1}(\Omega K)$ denote the integral class whose mod $p$ reduction is $\tilde{u}$. Thus $u_{2}$ is the $h^{s}$ image of $\tilde{u}$; this holds for all $s$, including $s=\infty$.

For $r+1 \leq s \leq 2 r$ we also have $u_{3}=[b,[b, a]]$ as a generator of $E^{s}(\Omega K)$. Put $\tilde{u}_{3}=[\tilde{b},[\tilde{b}, \tilde{a}]] \in \pi_{6 n-1}\left(\Omega K ; \mathbb{Z}_{p^{r}}\right)$. The fact that its " $p^{r}$-Bockstein," i.e., $3[\tilde{a},[\tilde{b}, \tilde{a}]] \in \pi_{6 n-2}\left(\Omega K ; \mathbb{Z}_{p^{r}}\right)$, is zero (because $\left.[\tilde{a}, \tilde{a}]=0\right)$ means that $\tilde{u}_{3}$ extends over $P^{6 n-1}\left(p^{2 r}\right)$, whence $\tilde{u}_{3}$ reduces $\bmod p$ to an element $\tilde{\tilde{u}}$ that survives to $E_{(\pi)}^{2 r}$. Since $h^{r}(\tilde{\tilde{u}})=u_{3}$ in $E^{r}$, we see that $u_{3} \in \operatorname{im}\left(h^{s}\right)$ for $r+1 \leq$ $s \leq 2 r$.

The remaining generator, which occurs in $E^{s}$ for $r+1 \leq s \leq \infty$, is $v_{1}$. To see that $v_{1}$ belongs to $\operatorname{im}\left(h^{s}\right)$, first note that $\pi_{8 n-2}(\Omega K)$ is a rank one abelian group; denote an infinite cyclic generator by $v^{*}$. In rational homotopy $K$ is merely $S^{4 n}$, which has a nontrivial Whitehead square, so we know that

$$
\left[u^{*}, u^{*}\right]=k v^{*}+w^{*} \text { in } \pi_{8 n-2}(\Omega K),
$$

where $k \neq 0$ and $w^{*}$ is a torsion element. Applying the Hurewicz homomorphism $h$ to $(18)$ gives

$$
2 u_{2}^{2}=h\left(\left[u^{*}, u^{*}\right]\right)=k h\left(v^{*}\right)+h\left(w^{*}\right)
$$

in $H_{*}(\Omega K)=H_{*}(A, d)$. Since $v_{1}$ generates an infinite cyclic summand of $H_{8 n-2}(A, d)=H_{8 n-2}(\Omega K)$, we may write

$$
h\left(v^{*}\right)=k^{\prime} v_{1}+v_{2}
$$

where $v_{2}$ is a torsion element of $H_{8 n-2}(A, d)$. A direct calculation in $A$ reveals that

$$
2 p^{r}\left(u_{2}^{2}\right)=2 p^{2 r} v_{1}+d\left([[[a, b], b], b]+2 p^{r}[[c, b], b]\right),
$$

whence $k k^{\prime}=2 p^{r}$. (In (20), $v_{1}$ refers to the integral cycle $p^{r} c^{2}+2[[c, a], b]$, whereas in (19) $v_{1}$ refers to the corresponding homology class.)

We will be done when we show that $k^{\prime}$ is a unit in $\mathbb{Z}_{(p)}$. This will follow, using $k k^{\prime}=2 p^{r}$, from showing $k$ to be divisible by $p^{r}$. To get there, we argue by contradiction. If $k$ were indivisible by $p^{r}$, then by (18) the mod $p^{r}$ reduction of $\left[u^{*}, u^{*}\right]$, which is the class $\left[\tilde{u}_{2}, \tilde{u}_{2}\right] \in \pi_{8 n-2}\left(\Omega K ; \mathbb{Z}_{p^{r}}\right)$, would be nonzero. Furthermore, because $k v^{*}$ is not a torsion element, $\left[\tilde{u}_{2}, \tilde{u}_{2}\right]$ would be nonzero in the quotient group $\pi_{8 n-2}\left(\Omega K ; \mathbb{Z}_{p^{r}}\right) / \operatorname{im}\left(\tilde{\beta}^{r}\right)$, where

$$
\tilde{\beta}^{r}: \pi_{8 n-1}\left(; \mathbb{Z}_{p^{r}}\right) \rightarrow \pi_{8 n-2}\left(; \mathbb{Z}_{p^{r}}\right)
$$

denotes the " $p^{r}$-Bockstein," i.e., composition on the right with

$$
P^{8 n-2}\left(p^{r}\right) \rightarrow S^{8 n-2} \rightarrow P^{8 n-1}\left(p^{r}\right) .
$$

However, we may compute directly that

$$
\tilde{\beta}^{r}([[[\tilde{a}, \tilde{b}], \tilde{b}], \tilde{b}])=[[\tilde{b}, \tilde{a}],[\tilde{b}, \tilde{a}]]=\left[\tilde{u}_{2}, \tilde{u}_{2}\right]
$$

for $\pi_{8 n-2}\left(\Omega K ; \mathbb{Z}_{p^{r}}\right)$ by using the relation $[\tilde{a}, \tilde{a}]=0$.

This argument shows $v_{1}$ in $E^{s}$ to belong to $\operatorname{im}\left(h^{s}\right)$ for all $s$ ( $v_{1}$ coincides with $2[e, b]$ when $s \leq r)$, and completes the proof.

From the above computation, the following information may readily be deduced. 
Conclusions. Let $K$ be as in (7), with $n \geq 1$ and $r \geq 1$ arbitrary but with $p \geq 5$. Localize away from the primes 2 and 3 . Then $\Omega K \in \Pi(\mathscr{S} \cup \mathscr{T})$. In particular,

$$
\Omega K \approx T^{2 n+1}\left\{p^{r}\right\} \times S^{4 n-1} \times S^{6 n-1}\left\{p^{2 r}\right\} \times \Omega S^{8 n-1} \times S^{8 n-1}\left\{p^{r}\right\} \times Y,
$$

where $Y$ is $(10 n-4)$-connected and $Y \in \prod \mathscr{T}(r, 2 r)$. Here $\mathscr{T}(r, 2 r)$ is the union of the four families of spaces: $S^{2 m+1}\left\{p^{r}\right\}, T^{2 m+1}\left\{p^{r}\right\}, S^{2 m+1}\left\{p^{2 r}\right\}$, and $T^{2 m+1}\left\{p^{2 r}\right\}$ for $m \geq 1$. Furthermore, at the prime $p$ the homotopy exponent of $K$ is $\max (2 r+1,4 n-1)$. Moore's conjecture holds for $K$.

Remark. The computation of the $p$-component of the homotopy groups of $K$ has now been reduced to three simpler problems: a combinatorial problem, an extension problem, and the homotopy groups of spheres. The combinatorial problem is to determine the number of factors of each type comprising the product $Y$. This can be done by a process similar to that done for $\Omega P^{m}\left(p^{r}\right)$ in [CMN1]; but if one actually wanted a numerical answer then a recursive algorithm would be used, peeling the factors one at a time off the Hilbert series for $E^{r}$ and for $E^{2 r}$. The relationship between $\pi_{*}\left(S^{2 m+1}\left\{p^{r}\right.\right.$ or $\left.\left.p^{2 r}\right\}\right)$ and $\pi_{*}\left(S^{2 m+1}\right)$ is well known, but the relationship between $\pi_{*}\left(T^{2 m+1}\left\{p^{r}\right.\right.$ or $\left.\left.p^{2 r}\right\}\right)$ and $\pi_{*}\left(S^{i}\right)$ could require solving the extension problem posed by (4).

Discussion. Besides its value as an example illustrating the connection with Ext algebra and dga technology, two good reasons may be offered for studying $\Omega K$. First, in [NS] Neisendorfer and Selick approach Moore's conjecture from the perspective of working up to larger spaces from smaller ones. They examine, and verify Moore's conjecture for, a variety of two- and three-cell complexes. The complex $K$ provides a natural next step in their program. Moore's conjecture does hold for $K$, but the fact that several pages of calculation were needed in order to establish this suggests that $K$ may already be near the borderline of complexity beyond which a direct individualized computation will become impractical.

As a second reason, the complex $K$ can act as a universal example. For an even-dimensional homotopy class $\alpha$ to have order $p^{r}$ and simultaneously to have its Whitehead square vanish does not seem unusual or far-fetched. If we are studying a space $X$ and we find an $\alpha \in \pi_{2 n}(X)$ with this property, then the map $\alpha: S^{2 n} \rightarrow X$ extends to $\tilde{\alpha}: K \rightarrow X$, and our understanding of $K$ may be useful for deducing information about $X$. The author originally undertook to analyze $\Omega K$ for precisely this reason, since it implied that $K$ would be an excellent "test case" for the Conjecture.

\section{REFERENCES}

[AH] J. F. Adams and P. J. Hilton, On the chain algebra of a loop spaces, Comment. Math. Helv. 30 (1955), 305-330.

[A1] D. J. Anick, A loop space whose homology has torsion of all orders, Pacific J. Math. 123 (1986), 257-262.

[A2] - On homotopy exponents for spaces of category two, Lecture Notes in Math., vol. 1370, Springer-Verlag, Berlin-New York, 1989, pp. 24-52.

[A3] _ Hopf algebras up to homotopy, J. Amer. Math. Soc. 2 (1989), 417-453.

[B] H. J. Baues, Commutator calculus and groups of homotopy classes, London Math. Soc. Lecture Note Ser., no. 50, Cambridge Univ. Press, Cambridge-New York, 1981. 
[BF] J. Backelin and R. Fröberg, Koszul algebras, Veronese subrings and rings with linear resolutions, Rev. Roumaine Math. Pures Appl. 30 (1985), 85-98.

[CMN1] F. R. Cohen, J. C. Moore, and J. A. Neisendorfer, Torsion in homotopy groups, Ann. of Math. (2) 109 (1979), 121-168.

[CMN2] _ Exponents in homotopy theory, Algebraic Topology and Algebraic $K$-Theory (W. Browder, ed.), Ann. of Math. Stud., no. 113, Princeton Univ. Press, Princeton, N.J., 1987, pp. 3-34.

[H] P. J. Hilton, On the homotopy groups of the union of spheres, J. London Math. Soc. 30 (1955), 154-172.

[J] I. M. James, Reduced product spaces, Ann. of Math. (2) 62 (1955), 170-197.

[L] C. Löfwall, On the subalgebra generated by the one-dimensional elements in the Yoneda Ext-algebra, Thesis, Report No. 5, Math. Inst., Stockholm Univ., Sweden, 1976.

[MW] C. McGibbon and C. Wilkerson, Loop spaces of finite complexes at large primes, Proc. Amer. Math. Soc. 96 (1986), 698-702.

[N1] J. A. Neisendorfer, Primary homotopy theory, Mem. Amer. Math. Soc., No. 232 (1980).

[N2] _ The exponent of a Moore space, Algebraic Topology and Algebraic $K$-Theory (W. Browder, ed.), Ann. of Math. Stud., no. 113, Princeton Univ. Press, Princeton, N.J., 1987, pp. 35-71.

[NS] J. A. Neisendorfer and P. Selick, Some examples of spaces with or without exponents, Canad. Math. Soc. Conf. Proc., vol. 2, Part 1, Amer. Math. Soc., Providence, R.I., 1982, pp. 343357.

[S] J.-P. Serre, Homologie singulière des espaces fibrés, Ann. of Math. (2) 54 (1951), 425-505.

[Sm] L. Smith, Split extensions of Hopf algebras and semi-tensor products, Math. Scand. 26 (1970), 17-41.

[W] C. Wilkerson, Genus and cancellation, Topology 14 (1975), 29-36.

Department of Mathematics, Massachusetts Institute of Technology, Cambridge, MASSACHUSETTS 02139 